\title{
Lack of compensatory increase in islet blood flow and islet mass in GK rats following $60 \%$ partial pancreatectomy
}

\author{
Annika M Svensson, Claes-Göran Östenson ${ }^{1}$, Birgitta Bodin and \\ Leif Jansson
}

\author{
Department of Medical Cell Biology, Uppsala University, Uppsala, Sweden \\ ${ }^{1}$ The Rolf Luft Centre for Diabetes Research, Department of Molecular Medicine, Endocrine and Diabetes Unit, Karolinska Institute and Hospital, \\ Stockholm, Sweden \\ (Requests for offprints should be addressed to Leif Jansson, Department of Medical Cell Biology, Biomedical Centre, Box 571 , SE-751 23 Uppsala, Sweden; \\ Email: Leif.Jansson@medcellbiol.uu.se) \\ (Annika M Svensson is currently at Department of Pathology and Laboratory Medicine, University of Vermont, Burlington, VT, USA)
}

\begin{abstract}
The effects of a $60 \%$ partial pancreatectomy were studied in hyperglycemic GK (Goto-Kakizaki) rats. Partial pancreatectomy or a sham operation was performed on 12-weekold female Wistar rats, GK rats or hybrids between male GK rats and female Wistar rats. Measurements of pancreatic blood flow and islet blood flow were performed by a microsphere technique 2 weeks after surgery. Glucose tolerance was decreased in hybrid compared with Wistar rats, and in GK rats compared with both hybrid and Wistar rats before surgery. Partial pancreatectomy induced minor changes in glucose tolerance. Wistar rats had a decreased islet mass following partial pancreatectomy. Both hybrid
\end{abstract}

and GK rats showed a significant decrease in relative islet volume, but only GK rats in total islet mass, compared with Wistar rats 2 weeks after surgery. Pancreatic blood flow and islet blood flow did not significantly differ between sham-operated Wistar, hybrid or GK rats. After partial pancreatectomy, islet blood flow in relation to islet mass increased 3-fold in Wistar rats and 2-fold in hybrid rats. In contrast, GK rats showed no increase in islet blood flow following partial pancreatectomy. It is concluded that compensatory mechanisms after partial pancreatectomy are operating less efficiently in hybrid and GK rats.

Journal of Endocrinology (2005) 184, 319-327

\section{Introduction}

The regulation of islet blood flow is distinct from that of the exocrine pancreatic blood flow. A number of possibly interdependent neural and local factors have been suggested to influence islet blood perfusion (Jansson 1994, Brunicardi et al. 1996). Our earlier investigations have pointed to an association between increased functional load on the islets and increased islet blood flow in different experimental systems, namely $48 \mathrm{~h}$ continuous highglucose load, partial pancreatectomy and diet-induced and inherited obesity (Jansson \& Sandler 1989, Styrud et al. 1992, Jansson 1994, Svensson et al. 1996). Although the mechanisms underlying these aberrations remain obscure, it could be envisaged that increased islet blood flow is a compensatory mechanism that may contribute to increased hormone output.

The GK (Goto-Kakizaki) rat is a non-obese animal model of hereditary non-insulin-dependent diabetes mellitus with mild hyperglycemia, initially derived from normal Wistar rats by repeatedly selecting animals with slightly aberrant glucose-tolerance tests for breeding (Goto
\& Kakizaki 1981, Kimura et al. 1982, Suzuki \& Goto 1987). The exact mechanism behind the impaired glucose-induced insulin release in the GK rat is unclear. However, it seems to be related to abnormalities in glucose metabolism and stimulus-secretion coupling within the $\beta$-cells (Giroix et al. 1993, Östenson et al. 1993a, 1993b, Hughes et al. 1994, MacDonald et al. 1996, Ling et al. 1998). $F_{1}$ hybrids between GK rats and Wistar rats are glucose-intolerant with impaired glucosestimulated insulin release (Abdel-Halim et al. 1994).

We have previously shown increased islet and pancreatic blood flow in normoglycemic rats following a 60\% pancreatectomy (Jansson \& Sandler 1989, 1991). This pancreatectomy procedure includes removal of the splenic part (tail region) of the pancreas, leaving the parts attached to the duodenum and stomach (duodenal region) intact, i.e. the regeneration of pancreatic tissue is observed exclusively in the duodenal region. The present study was designed to investigate the effects on islet mass and pancreatic and islet blood flow by a further increase in functional load on hyperglycemic GK and hybrid rats achieved by a similar reduction in $\beta$-cell mass. Furthermore, 
we addressed the issue of possible heterogeneity in islet mass and blood perfusion between the duodenal and splenic regions of the pancreas, to exclude regional contributions to the observed differences.

\section{Materials and Methods}

\section{Animals}

Female Wistar rats were purchased from B \& K Universal $\mathrm{AB}$ (Sollentuna, Sweden). Female GK rats and $\mathrm{F}_{1}$ hybrids, which were offspring of female Wistar and male GK rats, were from the Stockholm GK colony (Department of Endocrinology, Karolinska Hospital, Stockholm, Sweden), established in 1989 with breeding couples from Tohoku University School of Medicine, Sendai, Japan. Animals were 12 weeks old at the start of the experiment. All animals had free access to tap water and pelleted food, with the exception of a $12 \mathrm{~h}$ fast preceding the glucosetolerance tests (see below). All experiments were approved by the local animal ethics committee.

\section{Intraperitoneal glucose-tolerance test (i.p. GTT)}

An i.p.GTT was performed 2 days before partial pancreatectomy and again 2 days before the blood-flow measurements. Following an overnight fast, the animals were injected intraperitoneally with a $30 \%(\mathrm{w} / \mathrm{v})$ D-glucose solution $(2 \mathrm{~g}$ glucose $/ \mathrm{kg}$ body weight). Blood glucose concentrations were measured with test reagent strips (ExacTech; Baxter Travenol, Deerfield, IL, USA) from blood drawn from the cut tip of the tail immediately before and 10, 30, 60 and 120 min after glucose administration.

\section{Partial pancreatectomy}

The animals were anaesthetized with an intraperitoneal injection of ekviticine (a combination of pentobarbital and chloral hydrate). The splenic region of the pancreas, i.e. all parts of the pancreas unattached to the stomach or duodenum, was carefully dissected with cotton-tip applicators and removed. Any bleeding was controlled by diathermy. In previous experiments (Jansson \& Sandler 1989), the part of the pancreas removed by this procedure has been shown to constitute approximately $60 \%$ of the whole gland. In sham-operated animals, the splenic portion was mobilized gently using cotton-tip applicators but left in place.

\section{Measurements of blood flow}

This procedure has been described in detail in previous publications (see Jansson \& Hellerström 1983). Rats were anaesthetized with an intraperitoneal injection of thiobutabarbital (Inactin; Research Biochemicals, Natick, MA, USA; $120 \mathrm{mg} / \mathrm{kg}$ body weight), heparinized and placed on an operating table maintained at body temperature $\left(38^{\circ} \mathrm{C}\right)$. Polyethylene catheters were inserted into the ascending aorta via the right carotid artery and into the left femoral artery. The aortic catheter was connected to a pressure transducer (PDCR 75/1; Druck, Groby, Leicestershire, UK) to monitor mean arterial blood presure. After mean arterial blood pressure had been stable for at least $10 \mathrm{~min},(1 \cdot 5-3 \cdot 0) \times 10^{5}$ non-radioactive microspheres (diameter, $11 \mu \mathrm{m}$; NEN-Trac; DuPont Pharmaceuticals, Wilmington, DE, USA) were injected into the ascending aorta for $10 \mathrm{~s}$. An arterial blood sample was collected by free flow from the catheter in the femoral artery starting $5 \mathrm{~s}$ before the microsphere injection and continuing for a total of $60 \mathrm{~s}$. The withdrawal rate was determined by weighing the sample. Blood glucose was measured in a sample from the carotid catheter using test reagent sticks as above. Another arterial sample was drawn for measurement of serum insulin. The animals were killed and the pancreas and adrenal glands were removed, blotted, weighed and treated with a freeze-thawing technique to visualize the pancreatic islets and the microspheres (see below; Jansson \& Hellerström 1981). Samples from the duodenum and colon were treated in parallel. The pancreases from the sham-operated animals were divided into the duodenal region (approximately 40\% of the pancreas), corresponding to the parts of the pancreas adherent to the duodenum or stomach, and the splenic region, constituting the remainder of the pancreas (approximately $60 \%$ of the gland). Pancreases from partially pancreatectomized rats were treated in toto. The number of microspheres was determined in each organ and in the reference blood sample. The organ blood-flow values were calculated according to the formula $Q_{\text {org }}=Q_{\text {ref }} \times N_{\text {org }} / N_{\text {ref }}$ where $Q_{\text {org }}$ is organ blood flow $(\mathrm{ml} / \mathrm{min}), Q_{\text {ref }}$ is withdrawal rate of the reference sample $(\mathrm{ml} / \mathrm{min}), N_{\text {org }}$ is number of microspheres present in the organ and $N_{\text {ref }}$ represents the number of microspheres in the reference sample. The blood-flow values of the adrenals were compared and a difference of $<10 \%$ in blood flow between the glands confirmed adequate mixing of the microspheres into the circulation. The adrenal glands were of similar size in all animals.

\section{Measurements of pancreatic islet volume and mass}

Upon removal, the pancreas was divided into a duodenal part, consisting of the parts of the gland adhering to the duodenum or stomach, and a splenic part, corresponding to the remainder of the pancreas. The pancreas was divided into small pieces, each weighing approximately $25 \mathrm{mg}$. These pancreatic pieces were compressed between two object slides and stored at $-20^{\circ} \mathrm{C}$ (Jansson \& Hellerström 1981). The number of microspheres in these samples was then counted (see above) under a microscope equipped with both bright- and dark-field illumination. The visualization of the islets in these samples also enabled 
Table 1 Values are obtained 2 weeks after a $60 \%$ partial pancreatectomy or sham operation in female Wistar, GK or $F_{i}$ hybrids between GK and Wistar rats

Strain
Pancreatectomy
No. of animals

Body weight (g)

Before surgery

2 weeks after surgery

Pancreas removed at pancreatectomy $(\mathrm{mg})$

Pancreas weight (mg)

Blood glucose $(\mathrm{mM})$

Serum insulin $(\mathrm{ng} / \mathrm{ml})$

Mean arterial blood pressure $(\mathrm{mmHg})$

\begin{tabular}{ll} 
Wistar & Wistar \\
No & Yes \\
7 & 8 \\
\hline
\end{tabular}

$\begin{array}{cc}229 \pm 4 & 231 \pm 2 \\ 232 \pm 4 \dagger & 234 \pm 4 \\ - & 524 \pm 21 \\ 889 \pm 45 & 440 \pm 25^{* * *} \\ 5 \cdot 9 \pm 0 \cdot 3 & 6 \cdot 4 \pm 0 \cdot 2 \\ 2 \cdot 64 \pm 0 \cdot 46 & 2 \cdot 14 \pm 0 \cdot 31 \\ 120 \pm 5 & 122 \pm 4\end{array}$

\begin{tabular}{ll} 
Hybrid & Hybrid \\
No & Yes \\
7 & 7 \\
\hline
\end{tabular}

$\begin{aligned} 225 & \pm 7 \\ 227 & \pm 7 \\ - & - \\ 937 & \pm 42 \\ 8 \cdot 0 & \pm 0 \cdot 5 \S \S \\ 2 \cdot 42 & \pm 0 \cdot 16 \\ 120 & \pm 5\end{aligned}$

$\begin{aligned} 232 & \pm 4 \\ 237 & \pm 5 \\ 486 & \pm 18 \\ 537 & \pm 27^{* * *} \\ 8 \cdot 5 & \pm 0 \cdot 5 \S \S \\ 2 \cdot 55 & \pm 0 \cdot 30 \\ 121 & \pm 3\end{aligned}$

\begin{tabular}{ll} 
GK & GK \\
No & Yes \\
7 & 8 \\
\hline
\end{tabular}

$\begin{array}{cl}218 \pm 7 & 227 \pm 4 \\ 224 \pm 8 \dagger \dagger & 232 \pm 4 \\ - & 466 \pm 17 \\ 815 \pm 25 & 479 \pm 14^{* * *} \\ 9 \cdot 3 \pm 0 \cdot 3 \S \S \S & 12 \cdot 3 \pm 0 \cdot 3 \S \S \S \\ 2 \cdot 18 \pm 0 \cdot 26 & 1 \cdot 45 \pm 0 \cdot 26 \\ 123 \pm 3 & 124 \pm 3\end{array}$

Values are means \pm S.E.M. ${ }^{* *} P<0 \cdot 001$ compared with non-pancreatectomized rats of the same strain. $\S \S P<0 \cdot 01$ and $\S \S \S P<0 \cdot 001$ compared with the corresponding group of Wistar rats. $+P<0.05$ and $+\uparrow P<0 \cdot 01$ compared with the corresponding value before surgery.

us to estimate the pancreatic islet volume within the pancreatic regions, and thereby also the islet mass, by a point-counting method (Weibel 1979) adapted for use in freeze-thawed samples (Carlsson et al. 1996). The islet mass was then calculated assuming a similar density of the endocrine and exocrine parenchyma. Islets with a diameter less than $50 \mu \mathrm{m}$ were not analyzed with this method, since the visualization technique makes their idenitification difficult. The estimated islet mass was obtained by multiplying the islet volume with the total pancreatic weight.

\section{Measurement of serum insulin concentrations}

Blood samples were drawn from the tail vein of nonfasting animals. The samples were centrifugated and the serum portion was separated and stored at $-20^{\circ} \mathrm{C}$. The insulin content was determined with ELISA (Insulin ELISA; Mercodia AB, Uppsala, Sweden) using rat insulin (Novo Nordic, Bagsværd, Denmark) as a standard.

\section{Statistical analysis}

All values are expressed as means \pm S.E.M. Statistical comparisons were made with Student's paired $t$-test or repeated-measurement ANOVA, with Bonferroni's correction (Sigmastat; SSPD, Erlangen, Germany).

\section{Results}

\section{Body weight and mean arterial blood pressure}

Before surgery there were no significant differences in body weight between Wistar, hybrid and GK rats (Table 1). A slight increase in body weight was seen 2 weeks following the procedure in sham-operated Wistar and GK rats, while no significant increase was seen in any of the other groups (Table 1). Mean arterial blood pressure (Table 1) and hematocrit values (results not shown) were similar in all animals.

\section{Pancreatic wet weight}

Sham-operated Wistar, hybrids and GK rats had similar total pancreatic wet weights (Table 1). Likewise, partially pancreatectomized Wistar, hybrids and GK rats had similar pancreatic weights 2 weeks after surgery. As expected, the weight of the pancreas was markedly lower in all animals subjected to a partial pancreatectomy compared with sham-operated rats of the same strain.

\section{Glucose tolerance (Fig. 1)}

Intraperitoneal glucose tolerance tests were performed 2 days before partial pancreatectomy and again 2 days

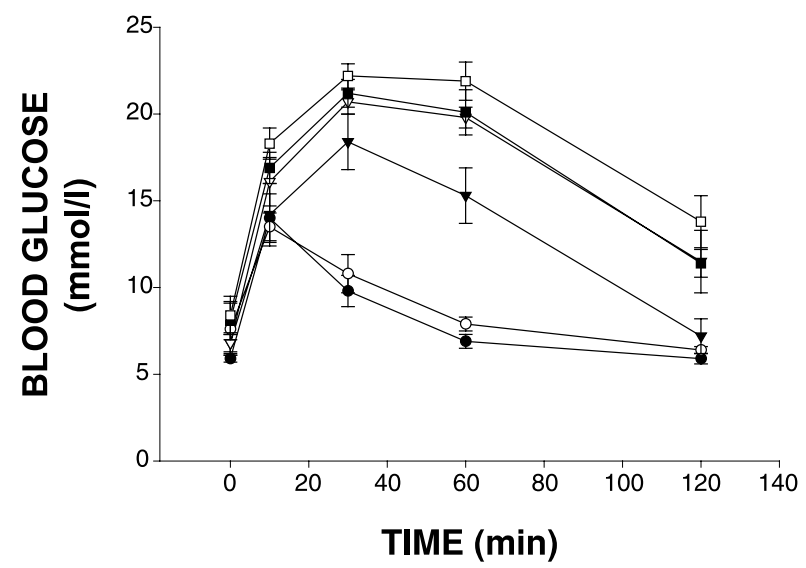

Figure 1 Blood glucose concentrations after an ipGTT (2 g/kg body weight) 12 days after a $60 \%$ partial pancreatectomy (open symbols) or sham operation (filled symbols) on female Wistar (circles), $\mathrm{F}_{1}$-hybrid (triangles) or GK (squares) rats. Values are means \pm S.E.M. from seven or eight experiments. 
before blood-flow measurements. Both hybrids and GK rats had impaired glucose tolerance compared with Wistar rats both before and after surgery. After partial pancreatectomy, there was a slight deterioration in glucose tolerance in Wistar rats $(P<0.05$ for blood glucose concentrations at 30 and $60 \mathrm{~min})$ and in hybrids $(P<0.05$ at $60 \mathrm{~min}$ ) compared with sham-operated rats of the same strain. In GK rats however, the values obtained 2 days before surgery did not differ from those seen 12 days after surgery, i.e. no worsening of the glucose tolerance was imposed by the partial pancreatectomy.

\section{Blood glucose and serum insulin concentrations (Table 1)}

Blood glucose concentrations at the time of the blood-flow measurements were increased in both hybrids and GK rats compared with Wistar rats. However, there were no significant differences between sham-operated and partially pancreatectomized animals when comparisons were made within the respective strains. Serum insulin concentrations were similar in all groups.

\section{Relative islet volume density (Fig. 2) and estimated total islet mass (Fig. 3)}

There were no differences in relative islet volume densities when comparing sham-operated Wistar, hybrid and GK rats. The relative islet volume density 2 weeks after partial pancreatectomy was higher in Wistar rats when compared with sham-operated animals of the same strain. The relative islet volume density in partially pancreatectomized hybrid and GK rats was lower when compared with the corresponding value in Wistar rats, but did not differ from the values in sham-operated rats of the same strain. However, the relative islet volume density of both partially pancreatectomized hybrid and GK rats was lower than that of sham-operated Wistar rats.

When total islet mass was estimated in the whole pancreas 2 weeks after surgery a lower value was seen in sham-operated GK rats compared with sham-operated Wistar rats, whereas no differences were seen when hybrid and Wistar rats were compared. As expected, all partially pancreatectomized rats showed decreased total islet mass compared with sham-operated rats of the same strain when calculated per whole gland $(P<0 \cdot 001$ for all comparisons against sham-operated control rats).

Regional differences in islet volume density (Fig. 2) and islet mass (Fig. 3)

The duodenal and splenic regions of the pancreas were analyzed separately in sham-operated animals with respect to islet volume density and islet mass. The splenic portion of the pancreas showed higher islet volume density compared with the duodenal part in both Wistar and hybrid rats. In GK rats, however, no such difference was seen $(0 \cdot 1>P>0 \cdot 05$ with ANOVA). Furthermore, the islet

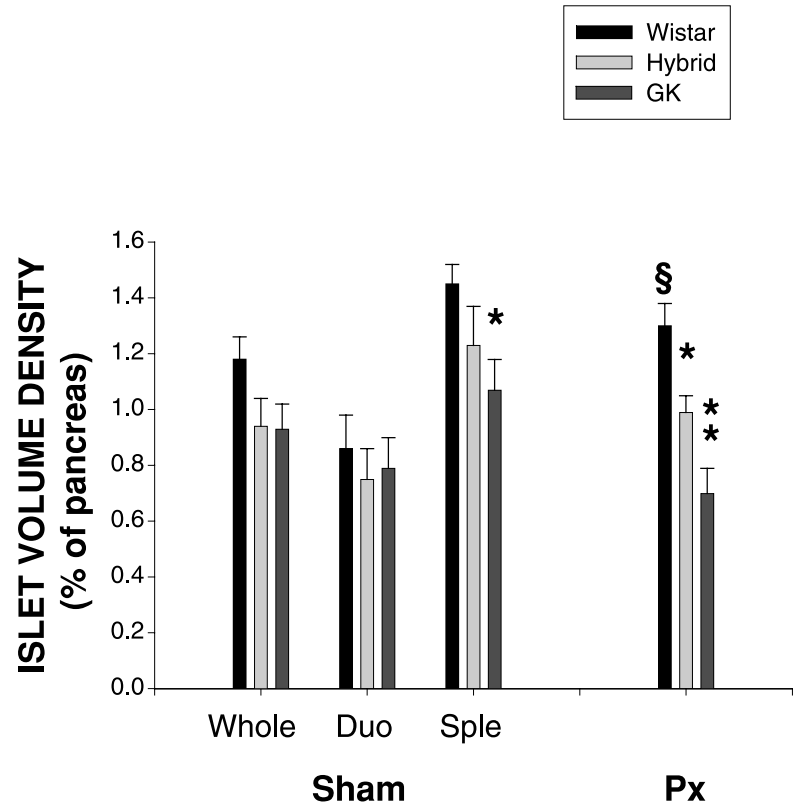

Figure 2 Islet volume density in the whole pancreas and in the duodenal and splenic regions of the pancreases in Wistar, $\mathrm{F}_{1}$-hybrid or GK rats 2 weeks after sham surgery or a $60 \%$ partial pancreatectomy $(\mathrm{Px})$. Values are means \pm S.E.M. from seven or eight experiments. ${ }^{*} P<0 \cdot 05$ and ${ }^{* *} P<0 \cdot 01$ when compared with the corresponding value in the whole pancreas of partially pancreatectomized Wistar rats. $\S P<0.05$ when compared with the corresponding value in sham-operated Wistar rats. Statistical comparisons were made with Student's paired $t$-test for comparisons between the duodenal (Duo) and splenic (Sple) region in the same strain, and with variance analysis for the other comparisons.

volume density in the splenic part of the pancreas was lower in GK rats compared with Wistar rats.

When islet mass was analyzed, a higher value was noted for the splenic portion compared with the duodenal part in all groups of sham-operated rats $(P<0.05$ for Wistar and hybrid rats and $P<0 \cdot 01$ for GK rats with ANOVA). The islet mass in the duodenal part of the pancreas of shamoperated rats was similar in all strains of animals. However, the islet mass in the splenic part of the gland in shamoperated rats was lower in GK rats when compared with the other strains.

\section{Total pancreatic blood flow (Fig. 4)}

Total pancreatic blood flow in Wistar rats, hybrids and GK rats was similar when sham-operated or partially pancreatectomized rats of the same strain were compared. Pancreatic blood flow was also measured in the duodenal and splenic parts of the gland in sham-operated rats, but no differences were seen when the different strains were compared. After partially pancreatectomy no differences between the strains were seen, even though there was a tendency towards a decrease in the GK rats $(P=0 \cdot 07)$. 


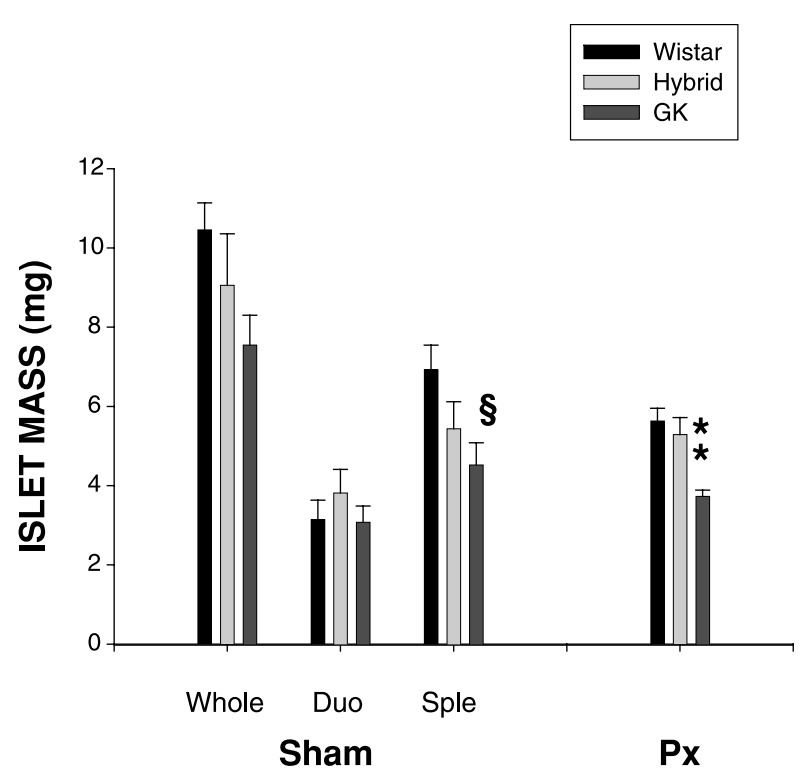

Figure 3 Islet mass in the whole pancreas and in the duodenal (Duo) and splenic (Sple) regions of the pancreases in Wistar, $\mathrm{F}_{1}$-hybrid or GK rats 2 weeks after sham surgery or a $60 \%$ partial pancreatectomy $(\mathrm{Px})$. Values are means \pm S.E.M. from seven or eight experiments. $\S P<0.05$ and ${ }^{* *} P<0.01$ when compared with the corresponding value for the other two strains as calculated with variance analysis.

\section{Islet blood flow (Fig. 5)}

Islet blood flow expressed per mg islet tissue was higher in Wistar rats compared with hybrid and GK rats $(P<0 \cdot 05$ for both comparisons; variance analysis). Furthermore, the blood perfusion of the islets in the splenic part of the gland of Wistar rats was almost twice as high as that to the duodenal part, whereas no such regional difference was seen in hybrid or GK rats. Partial pancreatectomy induced a pronounced increase in islet blood flow in Wistar rats, a less-marked augmentation in hybrid rats, and no change in GK rats when compared with sham-operated animals of the same strain.

When the blood perfusion of the whole islet organ, expressed as $\mu \mathrm{l} / \mathrm{min}$, was calculated, the flow values were similar in all groups of animals, with the exception of partially pancreatectomized GK rats, which had notably $(P<0 \cdot 001$ with ANOVA) lower blood-flow values (results not shown).

\section{Other regional blood-flow values (Table 2)}

There were no differences in colonic or duodenal blood flow between any of the groups. Adrenal blood flow was increased in sham-operated GK rats compared with Wistar rats, while the value for hybrid rats did not differ from that of the Wistar rats. Following pancreatectomy, an increase in adrenal blood flow was seen in the Wistar rats, while a decrease was demonstrated in GK rats.

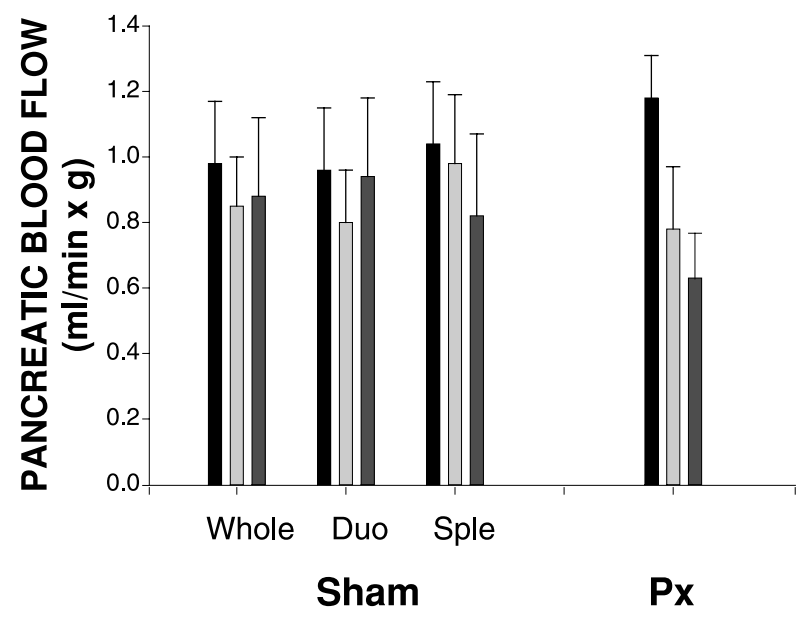

Figure 4 Pancreatic blood flow in the whole gland 2 weeks after a sham operation or a $60 \%$ partial pancreatectomy $(\mathrm{Px})$ in female Wistar, $F_{1}$-hybrid or GK rats. The blood-flow value is also provided separately for the duodenal (Duo) and splenic (Sple) portions of the pancreas in sham-operated rats. Values are means \pm S.E.M. from seven or eight experiments.

\section{Discussion}

The present study focused on the effects of increased demand for insulin secretion on pancreatic islet mass and blood flow following a reduction of functioning islets after partial pancreatectomy. Several models for partial obliteration of the $\beta$-cell mass are available. In addition to different protocols involving systemic administration of $\beta$-cell toxins, surgical techniques including 40,70 or $90 \%$ pancreatectomy have been described (see e.g. Pearson et al. 1977, Brockenbrough et al. 1988, Leahy et al. 1988, Lee et al. 1989, Jansson \& Sandler 1989, Tanigawa et al. 1996). Removal of $90 \%$ of the pancreas in normal rats results in greatly increased blood glucose levels, which may in turn affect the differentiation and function of $\beta$-cells per se (Jonas et al. 1999). Both the exocrine and endocrine tissue has been shown to regenerate $\beta$-cells by differentiation of duct precursor cells as well as by mitosis in pre-existing islets (Bonner-Weir et al. 1993). On the other hand, a $40 \%$ pancreatectomy does not lead to changes in glucose tolerance or glucose-induced insulin response 3 weeks after surgery (Leahy et al. 1988). Effects of a more pronounced reduction of the $\beta$-cell mass on insulin secretion have been evaluated previously in other animal models for glucose intolerance, and a decreased insulin response has been shown after increased 


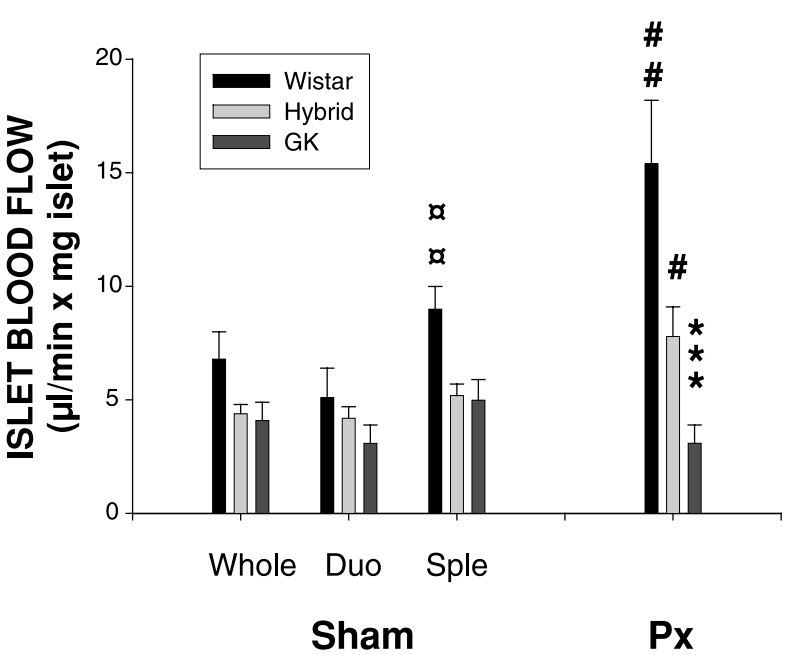

Figure 5 Islet blood flow 2 weeks after a sham operation or a $60 \%$ partial pancreatectomy (Px) in female Wistar, $\mathrm{F}_{1}$-hybrid or GK rats. The islet blood-flow value is also provided separately for the duodenal (Duo) and splenic (Sple) portions of the pancreas in sham-operated rats. Values are means \pm S.E.M. from seven or eight experiments. ${ }^{* *} P<0.001$ when compared with the corresponding value for sham-operated rats of the same strain and $a \mathbb{a} P<0.01$ when compared with the values for partially pancreatectomized Wistar and hybrid rats. ${ }^{\#} P<0.05$ and ${ }^{\# \#} P<0.01$ when compared with the corresponding value for the whole pancreas of the same strain. All comparisons were made with variance analysis.

load was applied (Leahy et al. 1988, Tanigawa et al. 1996).

In the present study a $60 \%$ pancreatectomy was performed. This can easily be accomplished by blunt dissection and removal of the splenic portion of the pancreas (for further details, see Jansson \& Sandler 1989). One inherent potential source of error with this model is that the only part that can be analyzed for regeneration following the procedure is the duodenal region, which has a relative lack of glucagon-producing $\alpha$-cells and a relatively high number of pancreatic polypeptide (PP) cells (Baetens et al. 1979). However, in several studies this has been shown to be of minor importance for growth and endocrine function (see Bonner-Weir 2000). In the present study we found that the splenic region contained more islets but had a blood perfusion similar to that of the duodenal region in control Wistar rats as well as hybrid rats, i.e. findings similar to those obtained previously in control rats (Jansson \& Hellerström 1987). No differences in either islet mass or blood perfusion between the two regions were seen in the GK rats. Thus, we deem this factor to be of minor importance for our findings.

In a preceding study, $60 \%$ pancreatectomy carried out in normoglycemic male Sprague-Dawley rats had only minor effects on glucose homeostasis (Jansson \& Sandler 1989). These results were confirmed in the present study, which showed unchanged serum insulin concentrations and minor changes in ipGTT in the normal Wistar rats following pancreatectomy. Also in hybrid rats the changes in glucose tolerance were of a small magnitude, while virtually no effects were noted in the GK rats.

Previous reports have shown progressive proliferation of fibrous tissue in the islets of GK rats, ultimately leading to the formation of irregularly shaped, so-called starfish islets, with a decreased number of $\beta$-cells (Goto \& Kakizaki 1981, Höög et al. 1996, 1997). However, in previous investigations involving 8-week-old Wistar, GK and hybrid rats from the Stockholm colony, a majority of the islets appeared histologically normal and only a few starfishshaped islets were found (Abdel-Halim et al. 1994, Guenifi et al. 1995). In other studies, no differences in islet $\beta$-cell density or insulin content were seen between GK rats and Wistar rats at 12 weeks of age (Ohneda et al. 1993, Höög et al. 1996, 1997, Metz et al. 1999). The animals studied in the present experiments were 14 weeks old, and were thus not expected to show significant aberrations in islet architecture or $\beta$-cell mass. Although the islet mass was found to be slightly lower in the sham-operated GK rats compared with Wistar and hybrid rats, the relative islet volumes were similar in all groups of sham-operated animals. Thus, although the $\beta$-cell mass may be slightly reduced in the GK rats, this change would not explain the metabolic aberrations in these animals. It should be noted that, in contrast to our findings, rats from the Paris colony of GK rats have demonstrated a pronounced decrease in islet volume at a very early age (Movassat et al. 1995). It could be assumed that such a decrease contributes to the glucose intolerance in these animals (Giroix et al. 1993).

Table 2 Organ blood flow values 2 weeks after a $60 \%$ partial pancreatectomy or sham operation in female Wistar, GK or $F_{i}$ hybrids between GK and Wistar rats

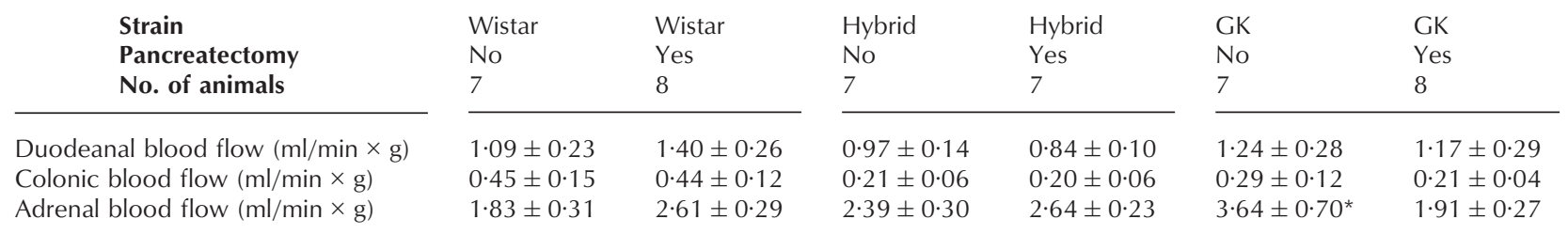


Following partial pancreatectomy, a marked reduction in islet mass was seen in all groups of rats. When pancreatectomized rats were compared, GK rats had significantly lower islet mass than both Wistar and hybrid rats. Taken together, our results suggest that after a $60 \%$ partial pancreatectomy Wistar rats are able to functionally compensate for the loss of insulin-producing tissue. In contrast, GK rats do not seem to be able to respond to the same degree, as reflected not only in the higher blood glucose concentrations of these animals, but also by a decrease in relative islet volume and a decreased islet mass compared with Wistar rats following pancreatectomy. This may indicate a relative impairment of the $\beta$-cell replication (see Brockenbrough et al. 1988, Lee et al. 1989). However, replication per se has not been evaluated in the present study. As expected (see Hellman 1959, Saito et al. 1978, Hellerström et al. 1988), islet cell mass was higher in the splenic portion of the pancreas in Wistar rats. This pattern of distribution was also present in hybrid and GK rats.

Previous studies have shown that a $60 \%$ pancreatectomy induces a marked increase in total pancreatic and islet blood perfusion during the first four post-operative weeks (Jansson \& Sandler 1989). In the present study, we chose to study the animals 2 weeks after surgery, i.e. when the post-operative blood-flow increase reaches a maximum (Jansson \& Sandler 1989) and there is still ongoing regeneration of the islets (Pearson et al. 1977, Brockenbrough et al. 1988).

Our previous study, using male Sprague-Dawley rats (Jansson \& Sandler 1989), showed an increase in total pancreatic blood flow 2 weeks post-surgery. In contrast, the female Wistar rats in the present study did not attain an increased pancreatic blood flow $(0 \cdot 05<P<0 \cdot 1)$. This inconsistency may be due to strain or sex differences.

Following partial pancreatectomy, a 2-fold increase in islet blood flow, as calculated in relation to islet volume, was seen in the Wistar rats. Thus, the islet blood flow in partially pancreatectomized Wistar rats increased to such a magnitude that the total blood perfusion to islets in these rats reached the same level as the islet blood flow in the whole pancreas in the sham-operated animals. This finding confirms previous observations linking augmented islet blood flow to settings with increased need for insulin secretion (see Jansson \& Sandler 1989, Styrud et al. 1992, Atef et al. 1992, 1994, Svensson et al. 1996). Obviously, increased islet blood flow could constitute one mechanism for maintaining normal glucose homeostasis in the face of increased functional demand, i.e. a sudden decrease in islet mass. The physiological and/or metabolic events underlying the increase in islet blood flow under these circumstances are unknown. However, we believe that it could reflect an increased metabolism of the islets, since we have recently shown that this can lead to a pronounced increase in islet blood flow in other models, in which the major mediator of the response seems to be adenosine (Carlsson et al. 2002). It can also be speculated that growth factors involved in the regeneration of islets (see Nielsen et al. 1999) can influence islet blood flow, but this notion remains hypothetical.

We and others previously demonstrated increased islet perfusion in adult GK and hybrid rats compared with Wistar rats (Atef et al. 1994, Svensson et al. 1994, 2000, Carlsson et al. 1997). However, in the present study, no significant differences in islet blood perfusion between sham-operated Wistar and sham-operated hybrid or GK rats were seen. The reason for this is unclear. Since the sham operation involves mobilization of the splenic part of the pancreas, it could be envisaged that this procedure per se affects blood perfusion. We have previously noted a decrease in islet blood flow 1 week after intra-abdominal sham operations in Sprague-Dawley rats (Jansson \& Andersson 1997), which may reflect responses to changes in abdominal pressure or inflammatory reactions following laparotomy. GK and hybrid rats may be more sensitive to such events, in line with our previous observations that vagotomy and treatment with atropine decreases islet blood flow in hybrid rats to levels similar to those of normal Wistar rats (Svensson et al. 1996).

The most important finding in the current investigation is the decrease in islet blood flow following partial pancreatectomy seen in GK rats, after correction for differences in islet mass. This is in contrast to the normal Wistar rats which have a 3 -fold increase in their islet blood flow following surgery, while hybrid rats show a 2-fold increase. This suggests that whereas normal rats may compensate for the surgical removal of endocrine tissue by regeneration and hyperperfusion of the islets, these mechanisms are much less efficient, or even absent, in GK rats. It is of interest that a similar decrease in islet blood flow is seen in aging GK rats, which have an even more impaired glucose tolerance (Svensson et al. 2000). The present findings lend support to the notion that, in this model, a decrease in islet blood flow may precede the functional impairment of the islets. It may be speculated that an increased functional load is initially associated with an augmented islet blood perfusion, providing nutrients and means of delivery of the secreted hormones to sustain normal glucose tolerance. However, an associated capillary hypertension, as seen in GK rats (Carlsson et al. 1997) may cause endothelial and organ damage (see Zatz \& Brenner 1986, Toppee \& Gimbrone 1999), leading to changes in islet blood-flow regulation and decreased blood perfusion, ultimately resulting in impairment of nutrient and hormone transport. This could contribute to the glucose intolerance seen in some animal models of type 2 diabetes.

\section{Acknowledgements}

The expert technical assistance of Ms Astrid Nordin is gratefully acknowledged. 


\section{Funding}

The study was supported by grants from the Swedish Medical Research Council (72X-109, 19X-00034), the Juvenile Diabetes Research Foundation, the Swedish Diabetes Association, the EFSD/Novo Nordisk for Type 2 Diabetes Research Grant and the Family Ernfors Fund. The authors declare that there is no conflict of interest that would prejudice the impartiality of this scientific work.

\section{References}

Abdel-Halim SM, Guenifi A, Grill V, Luthman H, Efendic S \& Östenson C-G 1994 Impact of diabetic inheritance on glucose tolerance and insulin secretion in spontaneously diabetic GK-Wistar rats. Diabetes 43 281-288.

Atef N, Ktorza A, Picon L \& Pénicaud L 1992 Increased islet blood flow in obese rats: role of the autonomic nervous system. American Journal of Physiology - Endocrinology and Metabolism 262 E736-E743

Atef N, Portha B \& Pénicaud L 1994 Changes in islet blood flow in rats with NIDDM. Diabetologia 37 677-668.

Baetens D, Malaisse-Lagae F, Perrelet A \& Orci L 1979 Endocrine pancreas: three-dimensional reconstruction shows two types of islets of Langerhans. Science 206 1323-1325.

Bonner-Weir S 2000 Islet growth and development in the adult. Journal of Molecular Endocrinology 24 297-302.

Bonner-Weir S, Baxter LA, Schuppin GT \& Smith FE 1993 A second pathway for regeneration of adult exocrine and endocrine pancreas. A possible recapitulation of embryonic development. Diabetes 42 1715-1720.

Brockenbrough JS, Weir GC \& Bonner-Weir S 1988 Discordance of exocrine and endocrine growth after $90 \%$ partial panreatectomy in rats. Diabetes 37 232-236.

Brunicardi, FC, Stagner J, Bonner-Weir S, Wayland H, Kleinman R, Livingston E, Guth P, Menger M, McCuskey R, Intaglietta M, et al. 1996 Microcirculation of the islets of Langerhans. Diabetes $\mathbf{4 5}$ 385-392.

Carlsson P-O, Andersson A \& Jansson L 1996 Pancreatic islet blood flow in normal and obese-hyperglycemic (ob/ob) mice. American Journal of Physiology - Endocrinology and Metabolism 271 E990-E995.

Carlsson P-O, Jansson L, Östenson C-G \& Källskog Ö 1997 Islet capillary blood pressure increase mediated by hyperglycemia in NIDDM GK rats. Diabetes 46 947-952.

Carlsson P-O, Olsson R, Källskog Ö, Andersson A \& Jansson L 2002 Glucose-induced pancreatic islet blood flow increase in rats: an interaction between nervous and metabolic mediators. American Journal of Physiology - Endocrinology and Metabolism 283 E457-E464.

Giroix M-H, Vesco L \& Portha P 1993 Functional and metabolic perturbations in isolated islets from the GK rat, a genetic model of noninsulin-dependent diabetes. Endocrinology 132 815-822.

Goto Y \& Kakizaki M 1981 The spontaneous diabetes rat: a model of noninsulin dependent diabetes mellitus. Proceedings of the Japanese Academy Series B 57 381-384.

Guenifi A, Abdel-Halim SM, Höög A, Falkmer S \& Östenson C-G 1995 Preserved beta-cell density in the endocrine pancreas of young, spontaneously diabetic Goto-Kakizaki (GK) rats. Pancreas $\mathbf{1 0}$ 148-153.

Hellerström C, Swenne I \& Andersson A 1988 Islet cell replication and diabetes. In The Pathology of the Endocrine Pancreas in Diabetes, pp 141-170. Eds PJ Lefébvre \& DG Pipeleers. Berlin: Springer-Verlag.

Hellman B 1959 The effect of ageing on the number of islets in the rat. Acta Endocrinologica (Copenhagen) 32 78-91.

Höög A, Sandberg-Nordqvist AC, Abdel-Halim SM, Carlsson-Skwirut C, Guenifi A, Tally M, Östenson C-G, Falkmer
S, Sara VR, Efendic S, et al. 1996 Increased amounts of a high molecular weight insulin-like growth factor II (IGF-II) peptide and IGF-II messenger ribonucleic acid in pancreatic islets of diabetic Goto-Kakizaki rats. Endocrinology 137 2415-2423.

Höög A, Hu W, Abdel-Halim SM, Falkmer S, Qing L \& Grimelius L 1997 Ultrastructural localization of insulin-like growth factor-2 (IGF-2) to the secretory granules of insulin cells: a study in normal and diabetic (GK) rats. Ultrastructural Pathology 21 457-466.

Hughes SJ, Suzuki K \& Goto Y 1994 The role of islet secretory function in the development of diabetes in the GK Wistar rat. Diabetologia 37 863-870.

Jansson L 1994 The regulation of pancreatic islet blood flow. Diabetes and Metabolism Reviews 10 407-416.

Jansson L \& Hellerström C 1981 A rapid method of visualizing the pancreatic islets for studies of islet capillary blood flow using non-radioactive microspheres. Acta Physiologica Scandinavica 113 371-374.

Jansson L \& Hellerström C 1983 Stimulation by glucose of the blood flow to the pancreatic islets of the rat. Diabetologia 25 45-50.

Jansson L \& Hellerström C 1987 The blood flow to the islets of Langerhans in different regions of the rat pancreas. Proceedings of the Society for Experimental Biology and Medicine 185 474-477.

Jansson L \& Sandler S 1989 Pancreatic and islet blood flow in the regenerating pancreas after a partial pancreatectomy in adult rats. Surgery (St. Louis) 106 861-866.

Jansson L \& Sandler S 1991 Partial pancreatectomy in rats causes an impairment of the glucose-induced stimulation of pancreatic islet blood flow. Experientia 47 627-629.

Jansson L \& Andersson A 1997 The endocrine pancreas and induced portal hypertension in rats: preferential increase in pancreatic islet blood perfusion mediated by nitric oxide. Pancreas 14 280-284.

Jonas JC, Sharma A, Hasenkamp W, Ilkova H, Patane G, Laybutt R, Bonner-Weir S \& Weir GC 1999 Chronic hyperglycemia triggers loss of pancreatic beta cell differentiation in an animal model of diabetes. Journal of Biological Chemistry 274 14112-14121.

Kimura K, Toyota T, Kakizaki M, Kudo M, Takebe K \& Goto Y 1982 Impaired insulin secretion in the spontaneous diabetes rats. Tohoku Journal of Experimental Medicine 137 453-459.

Leahy JL, Bonner-Weir S \& Weir GC 1988 Minimal chronic hyperglycemia is a critical determinant of impaired insulin secretion after an incomplete pancreatectomy. Journal of Clinical Investigation 81 1407-1414.

Lee HC, Bonner-Weir S, Weir GC \& Leahy JL 1989 Compensatory adaptation to partial pancreatectomy in the rat. Endocrinology 124 1571-1575.

Ling ZC, Efendic S, Wibom R, Abdel-Halim SM, Östenson C-G, Landau BR \& Khan A 1998 Glucose metabolism in Goto-Kakizaki rat islets. Endocrinology 139 2670-2675.

MacDonald MJ, Efendic S \& Östenson C-G 1996 Normalization by insulin treatment of low mitochondrial glycerol phosphate dehydrogenase and pyruvate carboxylase in pancreatic islets of the GK rat. Diabetologia 45 886-890.

Metz SA, Meredith M, Vadakekalam J, Rabaglia ME \& Kowluru A 1999 A defect late in stimulus-secretion coupling impairs insulin secretion in Goto-Kakizaki diabetic rats. Diabetes 48 1754-1762.

Movassat J, Saulnier C \& Portha B $1995 \beta$-cell mass depletion precedes the onset of hyperglycaemia in the GK rat, a genetic model of non-insulin-dependent diabetes mellitus. Diabetes and Metabolism 21 365-370.

Nielsen JH, Svensson C, Galsgaard ED, Möldrup A \& Billestrup N 1999 Beta cell proliferation and growth factors. Journal of Molecular Medicine 77 62-69.

Ohneda M, Johnson JH, Inman LR, Chen L, Suzuki K, Goto Y, Alam T, Ravazzola M, Orci L \& Unger RH 1993 GLUT2 expression and function in beta-cells of GK rats with NIDDM. Dissociation between reductions in glucose transport and glucose stimulated insulin secretion. Diabetes 42 1065-1072. 
Östenson C-G, Abdel-Halim SM, Rasschaert J, Malaisse-Lagae F, Meuris S, Sener A, Efendic S \& Malaisse WJ 1993a Deficient activity of FAD-linked glycerophosphate dehydrogenase in islets of GK rats. Diabetologia 36 722-726.

Östenson C-G, Khan A, Abdel-Halim SM, Guenifi A, Suzuki K, Goto Y \& Efendic S 1993b Abnormal insulin secretion and glucose metabolism in pancreatic islets from the spontaneously diabetic GK rat. Diabetologia 36 3-8.

Pearson KW, Scott D \& Torrance B 1977 Effect of partial surgical pancreatectomy in rats. I. Pancreatic regeneration. Gastroenterology $72469-473$.

Saito K, Iwama N \& Takahashi T 1978 Morphometrical analysis on topographical difference in size distribution, number and volume of islets in the human pancreas. Tohoku Journal of Experimental Medicine 124 177-186

Styrud J, Eriksson UJ \& Jansson L 1992 A continuous 48-hour glucose infusion in rats causes both an acute and a persistent redistribution of the blood flow within the pancreas. Endocrinology 130 2692-2696

Suzuki K \& Goto Y 1987 A model of non-insulin-dependent diabetes mellitus: GK (Goto-Kakizaki) rats. Progress in Diabetes Mellitus Research 22 211-217.

Svensson AM, Östenson C-G, Sandler S, Efendic S \& Jansson L 1994 Inhibition of nitric oxide synthase by $\mathrm{N}^{\mathrm{G}}$-nitro-L-arginine causes a preferential decrease in pancreatic islet blood flow in normal rats and spontaneously diabetic GK rats. Endocrinology 135 849-853.
Svensson AM, Hellerström C \& Jansson L 1996 Diet-induced obesity and pancreatic islet blood flow in the rat: a preferential increase in islet blood perfusion persists after withdrawal of the diet and normalization of body weight. Journal of Endocrinology 151 507-511.

Svensson AM, Östenson C-G \& Jansson L 2000 Age-dependent changes in pancreatic islet blood flow in GK rats, an animal model of noninsulin-dependent diabetes mellitus. American Journal of Physiology-Endocrinology and Metabolism 279 E1139-E1144.

Tanigawa K, Xu G, Nakamura S, Kawaguchi M, Kato Y \& Tamura K 1996 Impaired compensatory adaption to B-cell mass reduction in young spontaneously hypertensive rats (SHR). Pancreas $\mathbf{1 2}$ $237-242$

Toppee JN \& Gimbrone Jr MA 1999 Blood flow and vascular gene expression: fluid shear stress as a modulator of endothelial phenotype. Molecular Medicine Today 5 40-46.

Weibel ER 1979 Practical methods for biological morphometry. In Stereological Methods, vol. 1, pp 101-161. Ed ER Weibel. London: Academic Press.

Zatz R \& Brenner BM 1986 Pathogenesis of diabetic microangiopathy: the hemodynamic view. American Journal of Medicine 80 443-453.

Received 11 October 2004 Accepted 25 November 2004 